\title{
Case management reduces drinking during pregnancy among high- risk women
}

\author{
Philip A. May ${ }^{1,2,3}$, Anna-Susan Marais ${ }^{3}$, J. Phillip Gossage ${ }^{2}$, Ronel Barnard ${ }^{3}$, Belinda Joubert ${ }^{3}$, Marise \\ Cloete $^{3}$, Natalie Hendricks ${ }^{3}$, Sumien Roux ${ }^{3}$, Annalien Blom ${ }^{3}$, Jeanetta Steenekamp ${ }^{3}$, Theresa Alexander ${ }^{3}$, \\ Romena Andreas ${ }^{3}$, Suzanne Human ${ }^{3}$, Cudore Snell ${ }^{4}$, Soraya Seedat ${ }^{3}$, Charles D. Parry ${ }^{3,5}$, Wendy O. Kalberg ${ }^{2}$, \\ David Buckley ${ }^{2}$, and Jason Blankenship ${ }^{2}$ \\ ${ }^{1}$ The University of North Carolina at Chapel Hill, Gillings School of Global Public Health, Nutrition Research Institute, Chapel Hill, NC, United \\ States \\ ${ }^{2}$ The University of New Mexico, Center on Alcoholism, Substance Abuse, and Addictions (CASAA), Albuquerque, NM, United States \\ ${ }^{3}$ Stellenbosch University, Faculty of Health Sciences, Department of Psychiatry, Tygerberg, Republic of South Africa \\ ${ }^{4}$ Howard University, School of Social Work, Washington, DC, United States \\ ${ }^{5}$ Alcohol and Drug Abuse Research Unit, Medical Research Council, Tygerberg, Republic of South Africa
}

\section{Abstract}

Aim: To estimate the efficacy of Case Management (CM) for women at high risk for bearing a child with Fetal Alcohol Spectrum Disorders (FASD).

Design: Women were recruited from antenatal clinics and engaged in 18 months of CM.

Setting: A South African community with a subculture of heavy, regular, weekend, recreational drinking and with high documented rates of FASD.

Participants: Forty-one women who were at high risk for bearing a child with FASD.

Measures: Statistical analysis of trends in drinking and other risk factors.

Findings: At intake, $87.8 \%$ of the women were pregnant, most had previous alcohol-exposed pregnancies, $67.5 \%$ reported that most or all of their friends drank alcohol, and 50.0\% had stressful lives. CM was particularly valuable for pregnant women, as statistically significant reductions in alcohol risk were obtained for them in multiple variables: total drinks on weekends after six months of CM $(p=.026)$ and estimated peak blood alcohol concentration (BAC) at six $(p<.001)$ and 18 months ( $p<.001)$. For participants completing 18 months of CM, AUDIT scores improved significantly by 6-month follow-up (from 19.8 to 9.7, $p=$ .000 ), and although there were increases at 12 and 18 months, AUDIT scores indicate that problematic drinking remained statistically significantly lower than baseline throughout CM. Happiness scale scores correlated significantly with reduced drinking in most time periods.

Conclusions: Making an enduring change in drinking behavior is difficult in this social setting. Nonetheless, CM provided by skilled and empathic case managers reduced maternal drinking at critical times, and, therefore, alcohol exposure levels to the fetus.

Fetal Alcohol Spectrum Disorders (FASD) are the leading known preventable forms of birth defects and developmental disabilities in many human populations (May et al., 2008). Specific sub-populations in the Western and Northern Cape provinces of the Republic of South Africa (ZA) have among the highest documented prevalence of Fetal Alcohol Syndrome (FAS) and FASD in the world (May et al., 2000; 2007; Urban et al., 2008;
Viljoen et al., 2005). FAS, the most severe diagnosis within the spectrum of FASD, is characterized by a unique pattern of facial features, physical growth retardation, and developmental delays. All are caused primarily by heavy exposure to alcohol in utero (Hoyme et al., 2005; Stratton, Howe, \& Battaglia, 1996). The interaction of alcohol with other maternal risk factors, such as nutrition, maternal age, childbearing history, and specific host and environmental

Correspondence : Philip A. May, UNC Nutrition Research Institute, 500 Laureate Way, Kannapolis, NC, United States, 28081, E-mail: philip_may@unc.edu. Telephone: 704-250-5002. Fax: 704-250-5001.

Financial support: This project was funded by the National Institute on Alcohol Abuse, and Alcoholism (NIAAA) Grants RO1 AA09440, R01 AA11685, and RO1/UO1 AA0115134, and the National Institute on Minority Health and Health Disparities (NIMHD).

Keywords: fetal alcohol spectrum disorders (FASD), prevention, case management, alcohol abuse 
conditions, affect pregnancy outcome, directly or possibly through epigenetic factors (May \& Gossage, 2011). To the degree that maternal drinking and related co-factors of risk occur, various outcomes and diagnoses ensue: from children who are severely dysmorphic and behaviorally challenged (FAS and Partial FAS [PFAS]) to children with less dysmorphia and with cognitive and behavioral symptoms that are often less clear-cut in their manifestations and diagnoses (Alcohol-Related Neurodevelopmental Deficits [ARND]) and AlcoholRelated Birth Defects [ARBD]) (Stratton et al., 1996).

Because it is theoretically possible and practical to eliminate or reduce drinking among pregnant women through behavioral interventions, the United States Institute of Medicine (IOM) recommended comprehensive prevention of FASD at three levels: universal, selected, and indicated (Stratton et al., 1996). While universal methods such as public education and public policy are appropriate for entire populations, selected prevention techniques (e.g., advice on how to avoid alcohol before and during pregnancy) (Centre for Disease Control [CDC], 2005; Food and Drug Administration [FDA], 1981) are targeted to women of childbearing age. More specifically, indicated prevention of FASD is a tertiary-level approach where women of childbearing age who drink are provided birth control to prevent a pregnancy when they are drinking (Masis \& May, 1991; Velasquez et al., 2010). For women who are pregnant, or about to become pregnant, Case Management (CM) can be utilized to provide education, coaching, and support through a healthy pregnancy that is free from heavy drinking (Grant, Streissguth, \& Ernst, 2002; Grant et al., 2009; May et. al., 2008). CM, a major tool for FASD prevention, consists of a set of social service functions that helps women access their inner strengths and external resources in order to reduce alcohol use during pregnancy.

\section{Program Design and Theoretical Underpinnings}

CM was implemented by bilingual project officers-social workers or nurses who received two weeks of intensive, specialized training from experts in social work, Motivational Interviewing (MI), the Community Reinforcement Approach (CRA), and prevention. Most of the clinical training was carried out in the first language of the service population, Afrikaans, but some programmatic research and public health training was in English. Additionally, professional mentoring/coaching on consistent implementation of $\mathrm{CM}$ was provided in Afrikaans and English throughout the period of the CM program. Proven principles and methods of social work (Brun \& Rapp, 2001; Gursansky, Harvey, \& Kennedy, 2003; Rapp \& Goscha, 2004; Timberlake, Farber, \& Sabatino, 2002), MI (Miller \& Rollnick, 2002), and CRA (Meyers \& Smith, 1995) were used by case managers to encourage positive changes in lifestyle, childbearing practices, and drinking behavior.

Miller and Rollnick (2009) defined MI as a collaborative, person-centred method of guiding people to elicit and strengthen their motivation for change. The MI approach is respectful, quietly attentive, and supportive of the individual's right to make decisions and take action. Our application of this approach within CM is based on four key MI principles: expressing empathy through reflective listening; developing discrepancy in clients by drawing their attention to the negative impacts of their current behavior on their goals and values; rolling with client resistance to avoid arguments that undermine changes; and supporting self-efficacy by expressing optimism for change and highlighting a client's responsibility to choose and carry out changes.

CRA is a comprehensive, behavioral program that has been used successfully as a treatment approach for substance abuse problems. Its goal is to make a sober lifestyle more rewarding than the use of substances (Meyers \& Smith, 1995; Miller, Meyers, \& Hiller-Sturmhofel, 1999; Meyers, Smith, \& Lash, 2003). It involves the client's family members and friends, helping them to respond more effectively to high-risk behaviour and to reinforce the client's healthier choices (Herbeck, Hser, \& Teruya, 2008; Roozen et al., 2004).

\section{Methods}

\section{Recruitment of Subjects-Inclusion and Exclusion}

As part of a community-wide, comprehensive prevention program based on the IOM model of FASD prevention (Stratton et al., 1996), public education on FASD (pamphlets, videos, and discussion) and screening for drinking during pregnancy were provided by NIAAAfunded Stellenbosch University staff in government-funded community antenatal clinics. As depicted in Figure 1, inclusion in CM was specifically aimed at women who: (1) already had borne one child diagnosed with an FASD, or had drunk heavily during a previous pregnancy; or (2) had been drinking heavily during their current pregnancy (eight or more drinks per week, or one binge $\geq$ three drinks a day, any day of the week); or (3) scored high on the SelfAdministered Questionnaire (SAQ) (Bad Heart Bull, Kvigne, Leonardson, Lacina, \& Welty, 1999) or the Alcohol Use Disorders Identification Test (AUDIT) (Babor, de la Fuente, Saunders, \& Grant, 1992). If a woman met none of the above inclusion criteria (e.g., she was drinking at lower levels), then she was excluded from the study. The overarching, practical goals of CM were to protect the health of the fetus through prenatal care; to work with and support heavy-drinking pregnant women by motivating them to abstain or reduce their alcohol intake; and to contribute to a better life for these women by improving their day-to-day quality of life and therefore reducing the prevalence of FASD.

If women contacted in the antenatal clinics were found to be drinking heavily, or if they reported having done so during a past pregnancy, then they were screened further using the SAQ and/or the AUDIT. If an individual was found to be high risk, particularly if there was indication that a previous child of hers had been heavily exposed to alcohol, then she was invited to participate in CM on a regular basis for 18 months. 
Figure 1

Case management process

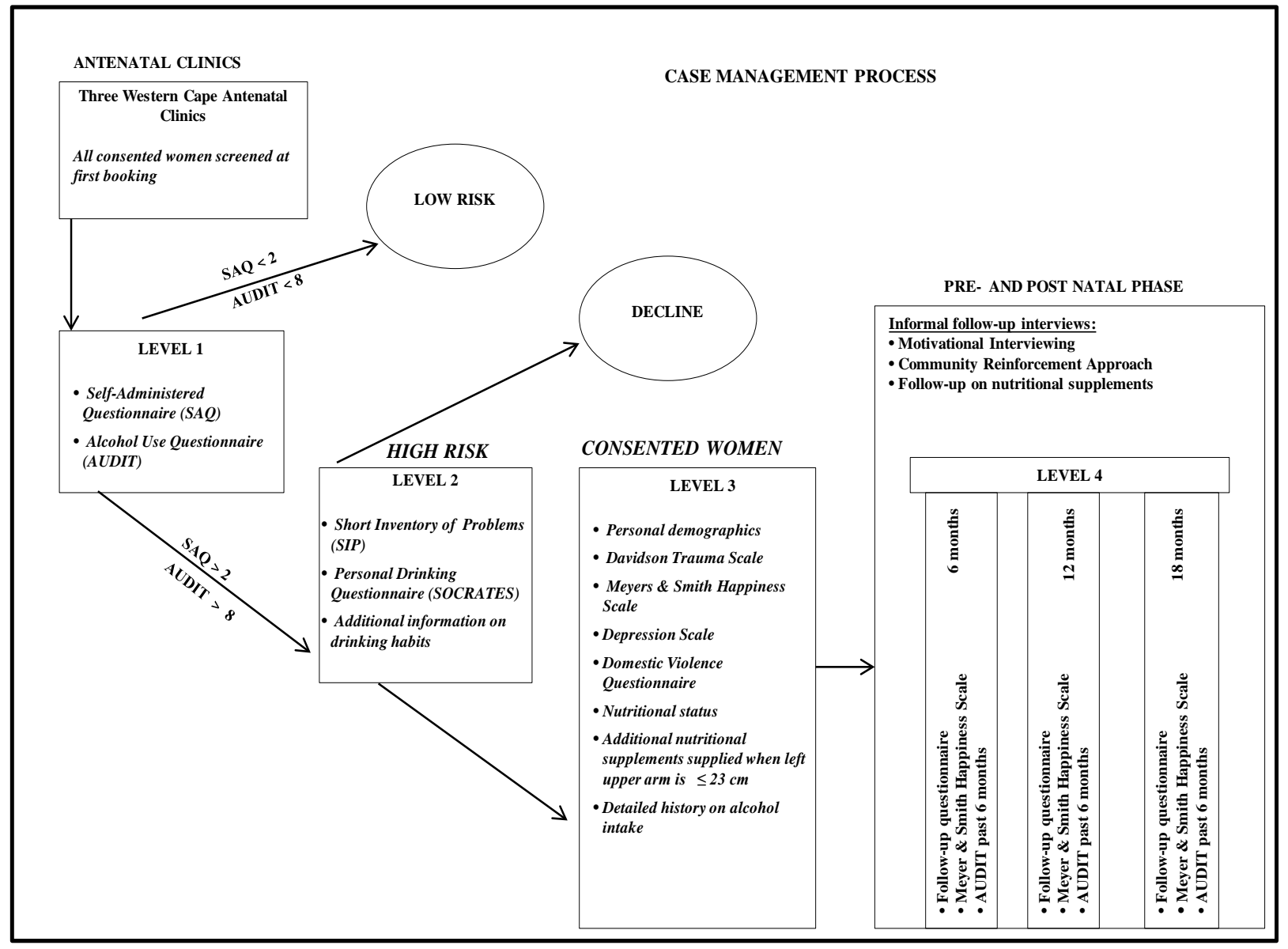

\section{Data Collection and Data Analysis}

Data were collected via interview at baseline, and 6, 12, and 18 months after participants entered CM. Items used to assess outcome over time included independent measures of quantity, frequency, timing and context of drinking, such as the number of drinks consumed per week and on weekends, and the number of drinks per day. The drinking problem scale used in this evaluation was the AUDIT (Babor et al., 1992). Scales assessing participant mental health were also used: the Happiness Scale (Meyers \& Smith, 1995) and Psychological Pain (Schneidman, 1999). Blood Alcohol Concentrations (BAC) were estimated by the BACCuS technique (Markham, Miller, \& Arciniega, 1993), which adjusts for the sex and weight of the person as well as the type of beverage, amount consumed, and duration of drinking episode.

Data analysis using SPSS Version 19 (SPSS, 2010) consisted of repeated measures analyses, with Bonferroni post hoc testing as appropriate, analyses of variance, t-tests, and basic cross-tabulation to examine the descriptive and material risk characteristics of the sample, and to examine change over time. Emphasis was placed on measures evaluating reduction or elimination of alcohol consumption during the index pregnancy.

\section{Results}

Over the first three-year course of this research project (up to February, 2012), 41 women participated. Of these women, 33 completed the 6-month follow-up, 31 completed the 12-month follow-up, and 30 completed the 18-month follow-up (see Figure 2). Thus, 11 (26.8\%) women began participation in the study but did not complete it. Table 1 indicates that the women who did not complete CM had relatively similar risk profiles to those women who remained in CM the entire 18 months. Of the women who dropped out, all but one were pregnant. Compared with the rest of the sample, dropouts were significantly lower risk in some ways (lower gravidity and parity), but higher risk in others (lower average Body Mass Index [BMI], a smaller head circumference, and a longer history of regular drinking) (see Table 1). At this point in 
Figure 2

Participation in case management over time

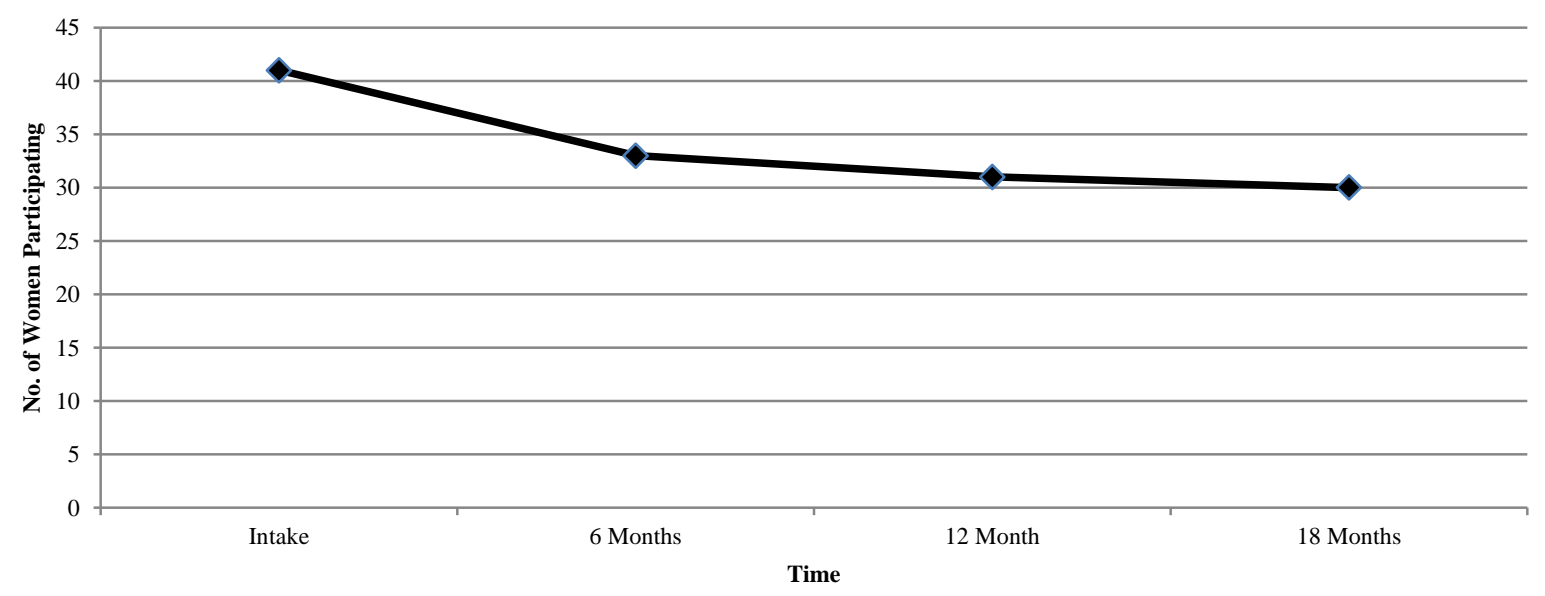

Table 1

Women who completed the study $(n=30)$ compared to women who dropped out $(n=11)$

\begin{tabular}{lcccc}
\hline Maternal Risk Variable & $\begin{array}{c}\text { Women who completed the } \\
\text { study Mean (SD) }\end{array}$ & $\begin{array}{c}\text { Women who dropped out of } \\
\text { the study Mean (SD) }\end{array}$ & $\boldsymbol{t}$ & $\boldsymbol{p}$ \\
\hline Age (years) & $24.9(5.7)$ & $24.5(5.3)$ & 0.20 & 0.842 \\
Gravidity & $2.7(1.6)$ & $1.4(0.5)$ & 2.78 & 0.012 \\
Parity & $2.1(1.2)$ & $0.6(0.5)$ & 3.84 & 0.002 \\
Height (cm) & $156.8(7.6)$ & $152.7(6.0)$ & 1.79 & 0.088 \\
Weight (kg) & $58.9(14.2)$ & $52.2(7.0)$ & 2.02 & 0.051 \\
Head circumference (cm) & $55.0(2.1)$ & $54.0(2.0)$ & 1.39 & 0.181 \\
BMI & $23.8(3.9)$ & $22.3(2.2)$ & 1.46 & 0.154 \\
Age of first drinking & $16.2(2.4)$ & $15.9(2.9)$ & 0.26 & 0.789 \\
Age of first drinking regularly & $17.3(2.6)$ & $16.1(2.5)$ & 1.33 & 0.201 \\
Number of years drinking regularly & $7.6(4.5)$ & $9.2(3.4)$ & -1.19 & 0.248 \\
\hline
\end{tabular}

the study, we do not know what forces were most influential on retention vs. departure.

Data (Table 2) indicate that the women in CM were in high-risk categories in terms of age at pregnancy (approaching age 25 and older) and high gravidity. Low BMI (25 or lower) is also indicative of high risk for producing affected offspring. The average age at which women in CM had started drinking was lower than indicated in community samples, and their total years of drinking regularly was higher (see May et al., 2005, 2008). Several of the drinking variables at baseline confirm that these women were most likely already concerned about their drinking, and had tried to reduce their alcohol consumption between the time they were first contacted at an antenatal clinic and their first CM visit, where baseline data were collected.
Although over $95 \%$ of the CM participants reported being Christian, nearly a third (28.9\%) reported never attending church (see Table 3). This may well be due to a problem of logistics for those who live and work on farms. Over 70\% of CM women did not work for money, and $87.8 \%$ were pregnant at the time of intake. The majority (67.5\%) of the sample reported that "half," "most," or "all" of their current friends drank alcohol. Half (50.0\%) of the women reported their lives to be "very" or "extremely" stressful.

\section{Drinking Characteristics}

Also in Table 2, the mean age of first drinking was 16.1 years and ranged from 10.0 years to 22.0 years of age. The mean duration of drinking (number of years that these women had consumed alcohol) was 8.0 years (minimum 1.0 years, maximum 18.0 years). At the time of the intake interview, the mean number of drinks per week was 5.1 drinks $(S D=9.1)$, and ranged from no drinks to 45.1 drinks per week. 
Table 2

Selected physical, childbearing and alcohol use data at intake for women in case management $(N=41)$

\begin{tabular}{|c|c|c|c|}
\hline Maternal Risk Variable & Mean $(S D)$ & Minimum & Maximum \\
\hline Age (years) & $24.8(5.6)$ & 15.0 & 39.0 \\
\hline Gravidity & $2.4(1.6)$ & 1.0 & 6.0 \\
\hline Parity & $1.7(1.2)$ & 0.0 & 4.0 \\
\hline Height $(\mathrm{cm})$ & $155.7(7.4)$ & 144.0 & 178.0 \\
\hline Weight (kg) & $57.1(12.9)$ & 40.0 & 110.0 \\
\hline Head circumference (cm) & $54.7(2.1)$ & 51.0 & 59.0 \\
\hline BMI & $23.4(3.6)$ & 17.4 & 34.7 \\
\hline Age of first drinking & $16.1(2.5)$ & 10.0 & 22.0 \\
\hline Age of first drinking regularly & $17.0(2.6)$ & 12.0 & 23.0 \\
\hline Number of years drinking regularly & $8.0(4.3)$ & 1.0 & 18.0 \\
\hline Percent abstinent at baseline in the last 7 days & $50.0 \%$ & & \\
\hline Percent abstinent at baseline in the last 30 days & $24.4 \%$ & & \\
\hline Percent abstinent at 6-month follow-up & $36.4 \%$ & & \\
\hline Total number of drinks over the past 7 days (total sample, baseline) & $5.1(9.1)$ & 0.0 & 45.1 \\
\hline Total number of drinks over the past 7 days (drinkers only, baseline) & $10.3(10.7)$ & 0.7 & 45.1 \\
\hline Total number of drinks over weekends (total sample, baseline) & $5.4(8.7)^{\mathrm{a}}$ & 0.0 & 40.7 \\
\hline Total number of drinks over weekends (drinkers only, baseline) & $10.6(10.0)$ & 0.7 & 40.7 \\
\hline AUDIT score (baseline) & $19.4(3.6)$ & 7.0 & 29.0 \\
\hline
\end{tabular}

${ }^{a}$ The fact that this value is greater than total number of drinks over the past 7 days is likely due to either memory or reporting error.

Table 3

Religious and social maternal risk variables and percent of women in case management responding

\begin{tabular}{|c|c|c|c|}
\hline \multirow{2}{*}{$\begin{array}{l}\text { Variable } \\
\text { Frequency of church attendance }\end{array}$} & \multicolumn{3}{|c|}{ Percent (\%) responding } \\
\hline & “Never” or “Not Very Often” = 52.6 & “Often” or "Very Often" = 47.3 & \\
\hline Do you work for money? & Yes $=26.8$ & No $=73.2$ & \\
\hline Occupation & Farm Worker $=42.5$ & Other occupation $=32.5$ & Not working $=25.0$ \\
\hline $\begin{array}{l}\text { Do you think you are pregnant } \\
\text { now? }\end{array}$ & Yes $=87.8$ & No $=12.2$ & \\
\hline How stressful is your life? & $\begin{array}{l}\text { "Not at all", "Somewhat", or } \\
\text { "Medium" = } 50.0\end{array}$ & “Very” or "Extremely” = 50.0 & \\
\hline \multirow[t]{2}{*}{$\begin{array}{l}\text { How many of your current friends } \\
\text { drink alcohol? }\end{array}$} & “None”, “Some”, or "Half” = 37.5 & “Most” or “All” = 57.5 & $\begin{array}{l}\text { "Does not have any } \\
\text { friends" }=5.0\end{array}$ \\
\hline & Standard drinks consumed per occasion & ver the past 30 days Mean (SD) & \\
\hline Job status: & Employed 4.4 (4.2) & Unemployed 3.9 (4.2) & \\
\hline
\end{tabular}

${ }^{\mathrm{a}}$ Most females recruited in antenatal clinics.

Most alcohol was consumed on weekends (Fridays, Saturdays, and Sundays), with a mean of 5.4 drinks ( $S D=$ 8.7), ranging widely from 0.0 drinks over the weekend to 40.7 drinks. In addition, over half the sample reported that "most" (15.0\%) or "all" (42.5\%) of their friends drank alcohol. Interestingly, there was a non-significant trend $(F$ $=.062, p=.805$ ) suggesting that persons who are employed consume more standard drinks per occasion $(\bar{x}=4.4, S D=$ $4.2)$ than those who are unemployed $(\bar{x}=3.9, S D=4.2)$.

Case managers indicate that those with more money can afford more alcohol. Repeated measures analyses of total drinks consumed over a weekend (see Figure 3) show no significant within-subjects main effect for either time $(F=$ $.159, p=.918)$ or time $\mathrm{x}$ pregnancy $(F=.423, p=.730)$. However, there is a significant between-subjects main effect: pregnant women consume significantly fewer drinks than do non-pregnant women in CM $(F=4.55, p=.043)$. Post hoc analyses indicate that at six months, pregnant women are consuming significantly less $(\bar{x}=2.7, t=3.62$, $p$ $=.026)$ than non-pregnant women $(\bar{x}=13.9)$.

\section{Drinking Characteristics During Pregnancy}

Estimated BAC's are presented from baseline to 18 months for pregnant and non-pregnant women in Figure 4. One 
participant's data were removed because of an erroneously excessive estimated BAC (e.g., Peak BAC > 8.000), leaving 29 subjects in this analysis. Although repeated measures analysis is not significant by time $(F=.256, p=$ .857) nor by the time $\mathrm{x}$ pregnancy interaction $(F=2.00, p=$ $.115)$, the between-subjects main effect of pregnancy is significant $(F=5.49, p=.022)$. Comparison between pregnant and non-pregnant women at six months shows significantly lower BAC for pregnant women during this critical time ( $t=-4.77, p=.000)$, as well as at 18 months ( $t$ $=3.67, p=.000)$. For those women who were pregnant, peak BAC standard deviations all went down, for all days (i.e., Friday, Saturday, and Sunday), at 6 months, indicating that heavier binges were reduced between baseline and 6 months.

The mean AUDIT score for the 29 women who were involved in CM for the entire 18 months was 19.4 at baseline ( $S D=6.7)$ and ranged from 7.0 to 29.0 (Figure 5). There was a significant drop in AUDIT scores to 9.7 at six months and a slight rise to 10.8 at 12 months and to 12.3 at 18 months. The top three AUDIT items (Figure 6) registering the largest reduction from baseline to six months were items 2, 3, and 5: "How many drinks containing alcohol do you have on a typical day when you are drinking?," "How often do you have six or more drinks on one occasion?," and "How often during the past six months have you failed to do what was normally expected from you because of drinking?”
The mean number of drinks per week prior to pregnancy was 20.0, ranging from 0.0 drinks to 87.5 drinks (see Table 4). Most women began $\mathrm{CM}$ in their third month of pregnancy. The mean number of drinks consumed per week in the second and third trimesters, and overall during the index pregnancy, is significantly lower (via paired $t$-test analysis) than the amount reported prior to pregnancy: 1st trimester was 19.3 drinks $(S D=17.4)$, when the women were usually not aware that they were pregnant; 2nd trimester was $10.4(S D=16.1)$; and third trimester was 3.7 drinks $(S D=14.1)$. Repeated-measures analysis also indicate that the overall within-subjects main effect of time is highly significant $(F=34.56, p=.000)$, with pairwise comparisons registering significant differences between number of drinks consumed per week prior to pregnancy and in the second trimester $(p=.000)$ and third trimester ( $p$ $=.000$ ). Likewise, there is a significant difference between number of drinks consumed per week in the first and second trimesters $(p=.000)$ versus the third $(p=.000)$ trimester. There is also a significant difference between the number of drinks consumed in the second and third trimester $(p=.001)$.

\section{Mental Health Characteristics-Psychological Pain and Happiness}

Half (50.0\%) of the women reported their lives to be either "very" (47.5\%) or "extremely" (2.5\%) stressful. Total Psychological Pain scores (adapted from Shneidman, 1999)

Figure 3

Total drinks consumed over a weekend at baseline and at 6, 12, and 18-month follow-ups ${ }^{a}$

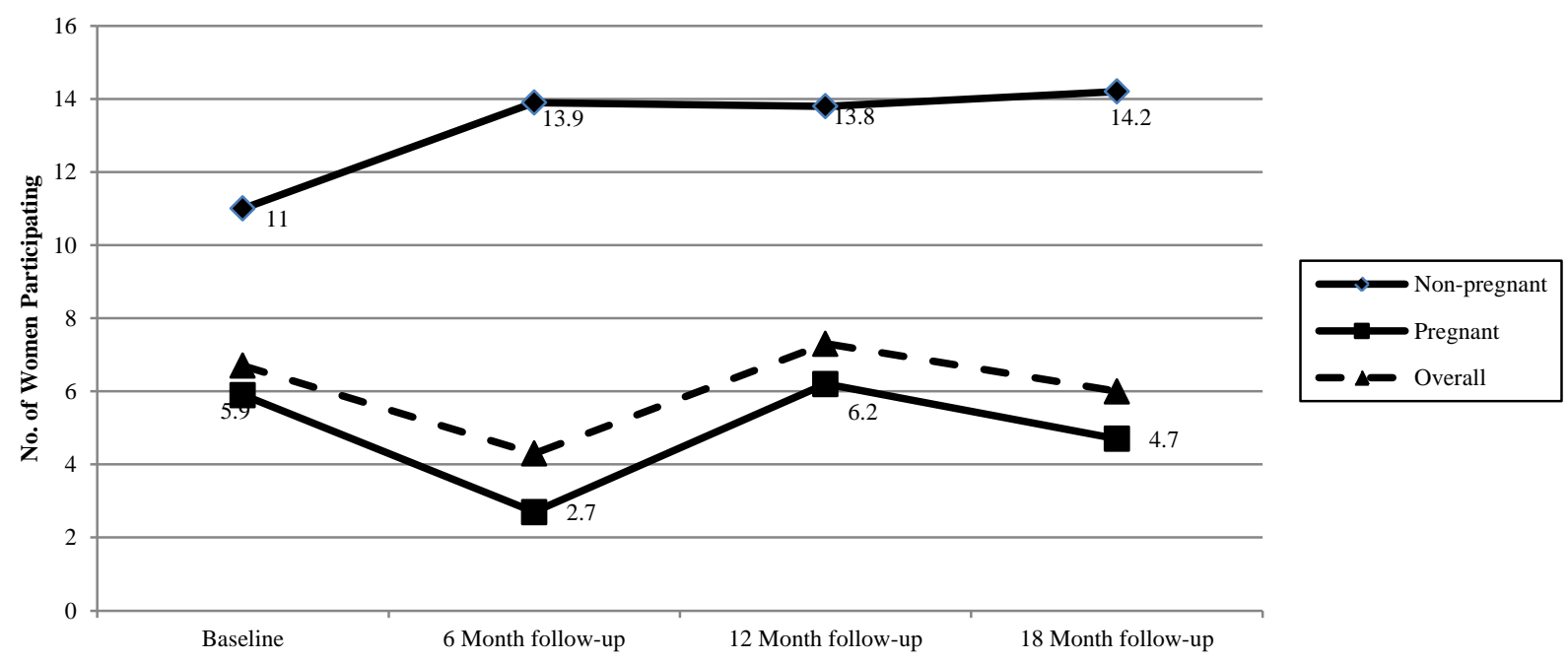

${ }^{\mathrm{a}}$ Data in Figure 3 include only those women who have data for all four time periods. ( $n=29$; pregnant; $n=25$, non-pregnant $n=4$, at baseline, 6 months, 12 months, and 18 months) Repeated measures analysis, between-Ss effect, pregnancy: $F=4.55, p=.043$

Repeated measures analysis, within-Ss effect, time: $F=.159, p=.918$

Repeated measures analysis, within-Ss effect, time x pregnancy: $F=.423, p=.730$

Pregnant vs. Non-Pregnant weekend drinking:

- $\quad$ At baseline, $t=.76, p=.486$

- At 6 months, $t=3.62, p=.026$

- At 12 months, $t=1.16, p=.275$

- At 18 months, $t=1.91, p=.120$ 


\section{Figure 4}

Estimated peak $B A C^{a}$ at baseline and at 6, 12, and 18-month follow-up for pregnant and non-pregnant women ${ }^{b}$

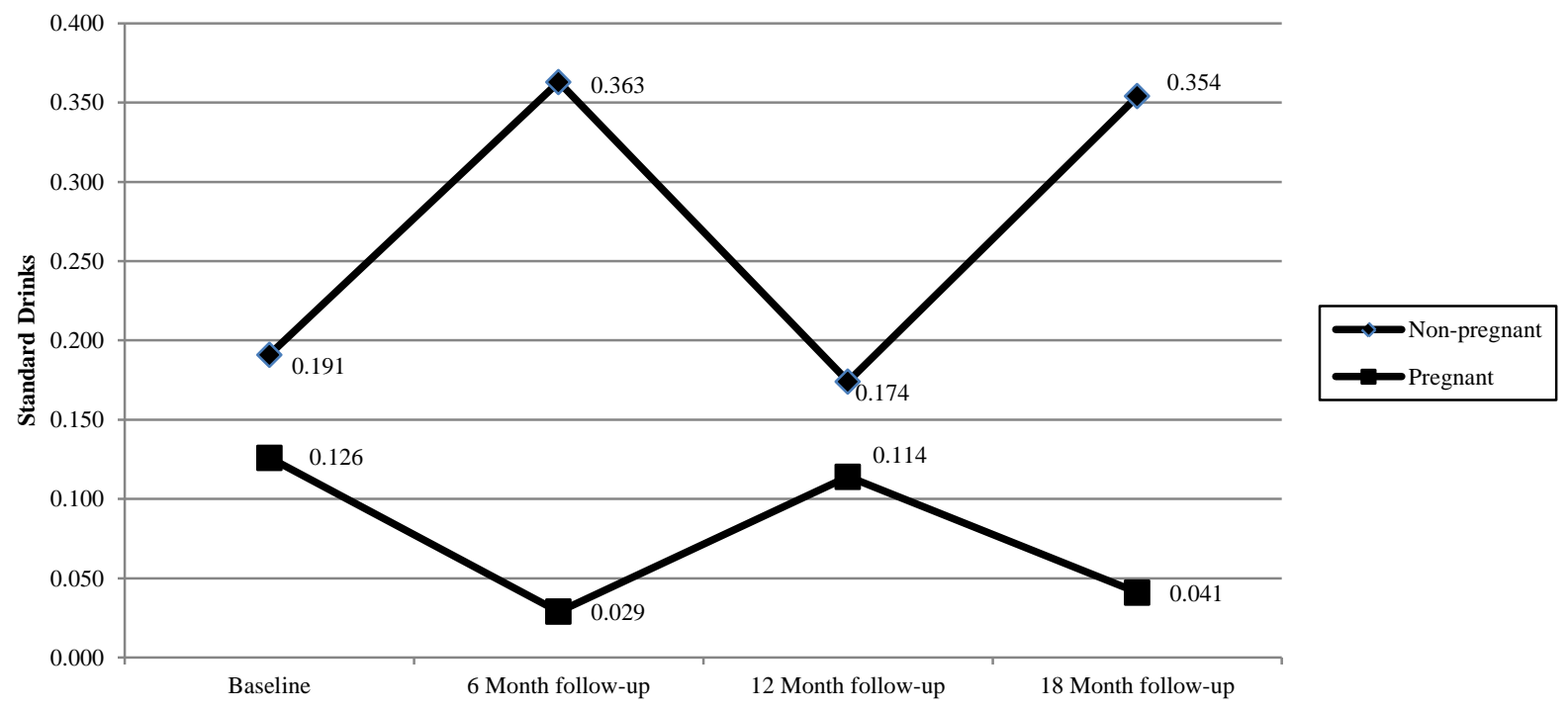

${ }^{a}$ Estimated by the BACCuS technique (Markham et al., 1993).

${ }^{\mathrm{b}}$ Data in Figure 4 include only those women who have data for all four time periods.

( $n=29$; pregnant, $n=25$, non-pregnant $n=4$, at baseline, 6 months, 12 months, and 18 months)

Repeated measures analysis, within-Ss effect, time, $F=.256, p=.857$

Repeated measures analysis, within-Ss effect, time $\mathrm{x}$ pregnancy, $F=2.00, p=.115$

Repeated measures analysis, between-Ss effect, pregnancy, $F=5.49, p=.022$

Comparison between Pregnant and Non-pregnant women:

- at Baseline, $t=-.57, p=.571$

- $\quad$ at 6 months, $t=-4.77, p=.000$

- $\quad$ at 12 months, $t=-.61, p=.541$

- $\quad$ at 18 months, $t=3.67, p=.000$

Figure 5

AUDIT score at baseline, 6, 12, and 18 month follow-up ${ }^{a}$

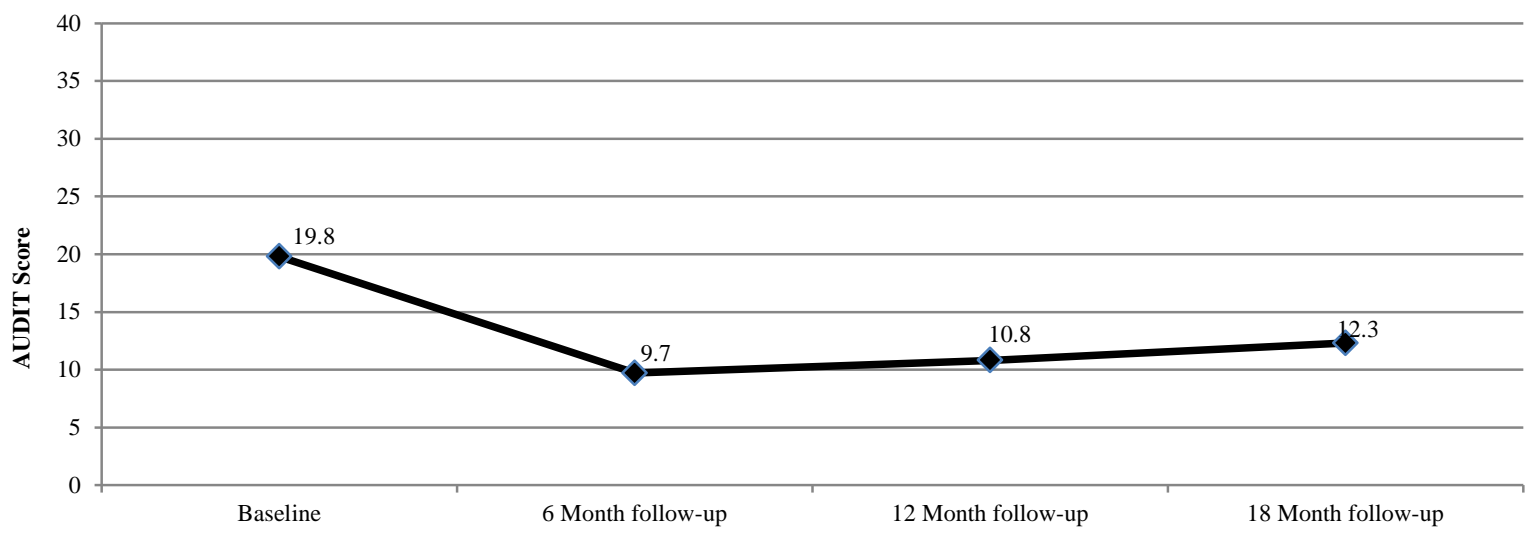

${ }^{a}$ Data in Figure 5 include only those women who have data for all four time periods.

( $n=29$ at Baseline, 6 months, 12 months, and 18 months)

Repeated measures analysis, within-Ss main effect: $F=14.26, p=.000$

Pairwise comparisons (Bonferroni):

- $\quad$ Baseline vs 6 month follow-up: $p=.000$

- $\quad$ Baseline vs 12 month follow-up: $p=.000$

- Baseline vs 18 month follow-up: $p=.000$ 


\section{Figure 6}

\section{AUDIT individual item scores by time $e^{a}$}

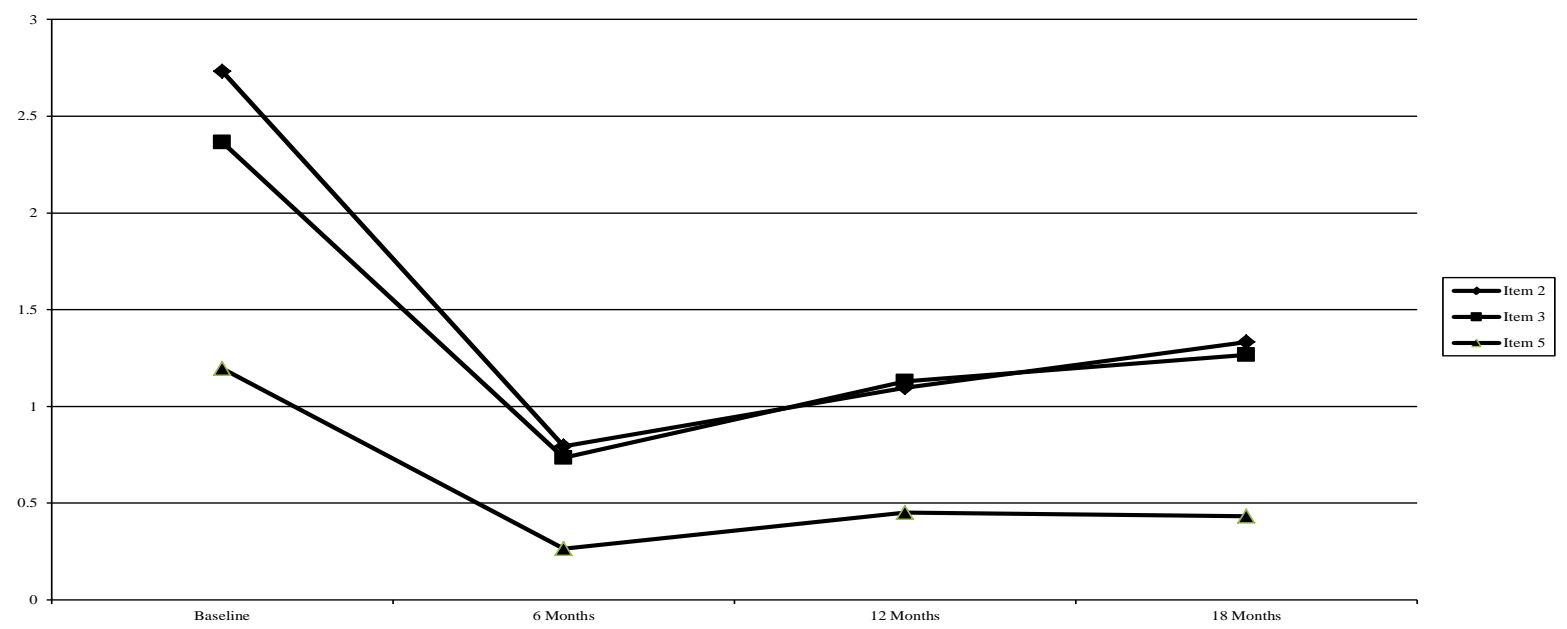

${ }^{\text {a }}$ Data in Figure 6 include only those women who have data for all four time periods. ( $n=30$ at Baseline, 6 months, 12 months, and 18 months)

Repeated measures analysis, within-Ss main effect, Time: $F=53.57, p=.000$

Repeated measures analysis, within-Ss main effect, Time x Audit Item: $F=2.19, p=.000$

Repeated measures analysis, between-Ss main effect, AUDIT Item, $F=13.36, p=.000$

The Top 3 Items showing the largest average difference from Baseline to 6 Months are:

Item 2: How many drinks containing alcohol do you have on a typical day when you are drinking?

Item 3: How often do you have six or more drinks on one occasion?

Item 5: How often during the past 6 months have you failed to do what was normally expected from you because of drinking?

\section{Table 4}

Standard drinks consumed before and during pregnancy

\begin{tabular}{lccc}
\hline Variable of Interest & Mean (SD) & $\boldsymbol{t}$ & $\boldsymbol{p}$ \\
\hline Number of drinks consumed per week prior to pregnancy & $20.0(17.1)$ & & 0.342 \\
Number of drinks consumed per week in the first trimester & $19.3(17.4)$ & 0.96 & 0.000 \\
Number of drinks consumed per week in the second trimester & $10.4(16.1)$ & 4.81 & 0.000 \\
Number of drinks consumed per week in the third trimester & $3.7(14.1)$ & 7.46 & 0.000 \\
Number of drinks consumed, per week, overall during pregnancy & $11.1(14.1)$ & 6.68 &
\end{tabular}

Note. Paired samples t-test score: comparing 'number of drinks consumed per week prior to pregnancy' versus the other variables of interest, by row

for the sample of women was approximately at the lower third of the scale $(\bar{x}=21.4, S D=12.8)$, although notes from the case managers conducting the interviews suggest that some women did not fully understand the concept of psychological pain. Nevertheless, a repeated measures analysis of Psychological Pain total score by time approached significance $(F=2.44, p=.072)$, showing a steady reduction in pain from baseline $(\bar{x}=21.0)$ to 18 month follow-up $(\bar{x}=16.0)$.

General well-being at intake, as measured by the Happiness Scale (Meyers \& Smith, 1995), indicated a reasonable level of reported happiness among the sample of women $(\bar{x}=$ 97.0 out of 130 possible points, $S D=17.9$ ). More specifically, Happiness Scale data tell us that money management and drinking versus sobriety are the most worrisome areas of their lives. Though repeated measures analysis showed the total happiness score to not be significant ( $F=1.18, p=.325$ ), results were in the positive direction (higher scores, indicating greater happiness), rising from baseline $(\bar{x}=94.6)$ to 18 -month follow-up $(\bar{x}=$ 98.5). Happiness score data over time are positive and reflect well on the case management process and the reduced drinking that occurred at six months and 12 months. At six months, the Total Happiness Score was significantly correlated with less Friday drinking $(R=$ .379, $p=.032)$, but not with reduced drinking on Saturday $(R=-.279, p=.122)$ or Sunday $(R=-.093, p=.612)$. At 12 months, Total Happiness Score was significantly correlated with less drinking on Friday $(R=-.571, p=$ $.002)$ and Saturday $(R=-.646, p=.000)$, but not Sunday $(R$ $=-.276, p=.155)$. Total Happiness Score at 18 months was not significantly correlated with any drinking data. 


\section{Discussion}

CM instituted in this high-risk community does reduce risk to the fetus by reducing levels of exposure to alcohol in pregnant women as measured by: AUDIT score, average alcohol consumption, and estimated peak BAC measures. Therefore, unborn children/fetuses benefited directly from reduced drinking and the mothers' lower peak BAC during gestation. CM appears to increase levels of happiness in the participants, which correlates with reduced drinking. CM was not efficacious in producing changes in the women who were not pregnant. Once pregnancy was completed among the other women, long-term improvements were not maintained; the formerly pregnant women often returned to the heavier drinking pattern that is normative among drinking women in this subpopulation, although their postpartum drinking is at somewhat reduced levels.

\section{Limitations and Other Considerations}

One limitation of the study is that $26.8 \%$ of the women enrolled at baseline dropped out before the 18-month period of $\mathrm{CM}$ and the evaluation were completed. The comparison of non-completers to completers in Table 1 indicates more similarities than differences, but questions still remain: were those who dropped out much higher risk than others, and did any of the contact with CM have any effect on their drinking behavior? Because the longitudinal AUDIT score analysis was limited to those women who completed 18 months of $\mathrm{CM}$, the reduction in drinking problems reported here represents a valid reduction in risk.

A second limitation of this evaluation may be that the subjects were not randomly assigned to either treatment or a control condition. Random assignments could have been made and the results evaluated by case control methods, but this would contravene a true public health spirit for this comprehensive prevention program in a small community, and the ethics of such a design might be questioned. Our goal was to provide the maximum amount of benefit to the maximum number of people to help this community cope with the tremendous FASD problem uncovered by previous research. Therefore, we believe it was both ethical and practical to evaluate efficacy through change over time. However, there is a need for future studies that provide drinking data for a matched control group.

Third, some might say that this effort failed because complete abstinence was not achieved with most of the women throughout pregnancy. But our epidemiological research has shown repeatedly that many children who were exposed to substantial amounts of alcohol in the prenatal period can be born with normal functioning and without a diagnosis on the continuum of FASD (May \& Gossage, 2011). The measurements of success here indicate clearly that drinking, especially heavy, episodic (binge) drinking and high peak BAC's, were reduced significantly in pregnant women to levels that rendered a diagnosable FASD improbable in the majority of the children born to these mothers in these particular pregnancies. Our clinical research team members believe that the gold standard for prevention is the birth of a child with growth, development, and behavioral functioning within normal population parameters. If $\mathrm{CM}$ can help accomplish that, it is a success.

\section{Conclusions}

This study indicates that utilizing $\mathrm{CM}$ as a prevention method helps women eliminate or reduce their alcohol intake during pregnancy. Participants' problem drinking scores showed a significant improvement from baseline to their six-month follow-up (from 19.8 to 9.7, $p=.000$ ), and although their scores rose incrementally from the six-month follow-up to the 12 and 18-month follow-ups, overall their scores remained lower than at baseline. Measures of the quantity of drinking that occurred and the estimated BAC to which the fetuses were exposed for vitally important time periods in the pregnancies followed a generally similar and positive pattern. These results support the efficacy of case management for use with high-risk drinkers, while also showing the often transitory nature of improvements and the difficulty of making enduring personal changes in an environment where weekend drinking remains popular and is one of the only available forms of recreation.

\section{Acknowledgements}

This project was funded by the National Institute on Alcohol Abuse, and Alcoholism (NIAAA) Grants RO1 AA09440, R01 AA11685, and RO1/UO1 AA0115134, and the National Institute on Minority Health and Health Disparities (NIMHD). NIAAA officials have provided intellectual guidance for, participated in, and supported South African studies of FASD in a variety of ways since 1996. Our deepest thanks are extended to the political and school officials of the study community and to many individuals in the community who have graciously hosted and assisted in the research process over the years. We gratefully acknowledge the data entry performed with excellence by Ella Spillman. Jo Carothers assisted in the preparation and submission of this manuscript. Active consent for subjects to participate in all phases of this study of case management was obtained. Protocols and consent forms were approved by The University of New Mexico School of Medicine, HRRC \#96-209, and 06-199; Stellenbosh University, Faculty of Health Sciences, \#N06/07/129; and The University of Cape Town, \#101/2004U.

\section{References}

Babor, T. F., de la Fuente, J. R., Saunders, J., \& Grant, M. (1992). AUDIT: The Alcohol Use Disorders Identification Test: Guidelines for use in primary health care. Geneva, Switzerland: World Health Organization.

Bad Heart Bull, L., Kvigne, V., Leonardson, G. R., Lacina, L., \& Welty, T. K. (1999). Validation of a selfadministered questionnaire to screen for prenatal alcohol use in Northern Plains Indian women. American Journal of Preventive Medicine, 16, 240243. 
Brun, C., \& Rapp, R. C. (2001). Strengths-based case management: Individuals' perspectives on strengths and the case manager relationship. Social Work, 46, 278-288.

Centers for Disease Control and Prevention (CDC). (2005). Notice to readers: Surgeon General's advisory on alcohol use in pregnancy. Morbidity and Mortality Weekly Report March 11, 54, 229.

Food and Drug Administration (FDA). (1981). Surgeon General's advisory on alcohol and pregnancy. FDA Drug Bulletin, 11, 9-10.

Grant, T., Streissguth, A., \& Ernst, C. (2002). Benefits and challenges of paraprofessional advocacy with mothers who abuse alcohol and drugs and their children. Zero to Three, 23, 14-20.

Grant, T. M., Huggins, J. E., Sampson, P. D., Ernst, C. C., Barr, H. M., \& Streissguth, A. P. (2009). Alcohol use before and during pregnancy in western Washington, 1989-2004: Implications for the prevention of fetal alcohol spectrum disorders. American Journal of Obstetrics and Gynecology, 200, 278-278.

Gursansky, D., Harvey, J., \& Kennedy, R. (2003). Case management policies, practice and professional business. New York, NY, United States: Columbia University Press.

Herbeck, D. M., Hser, Y. I., \& Teruya, C. (2008). Empirically supported substance abuse treatment approaches: A survey of treatment providers' perspectives and practices. Addictive Behaviors, 33, 699-712.

Hoyme, H. E., May, P. A., Kalberg, W. O., Kodituwakku, P., Gossage, J. P., Trujillo, P. M., . . Robinson, L. K. (2005). A practical clinical approach to diagnosis of fetal alcohol spectrum disorders: Clarification of the 1996 Institute of Medicine Criteria. Pediatrics. 115, 39-47.

Markham, M. R., Miller, W. R., \& Arciniega, L. (1993). BACCuS 2.01: Computer software for quantifying alcohol consumption (A blood alcohol concentration calculating system). Behavior Research Methods, Instruments, and Computers, 25, 420-421.

Masis, K. B., \& May, P. A. (1991). A comprehensive local program for the prevention of fetal alcohol syndrome. Public Health Reports, 106, 484-489.

May, P. A., Brooke, L. E., Gossage, J. P., Croxford, J., Adnams, C., Jones, K. L., ... Viljoen, D. (2000). The epidemiology of fetal alcohol syndrome in a South African community in the Western Cape Province. American Journal of Public Health, 90, 1905-1912.

May, P. A., Brooke, L. E., Gossage, J. P., Snell, C., Hendricks, L., Croxford, J., . . Viljoen, D. L. (2005). Maternal risk factors for fetal alcohol syndrome in the Western Cape Province of South Africa: A population-based study. American Journal of Public Health, 95, 1190-199.

May, P. A., \& Gossage, J. P. (2011). Maternal risk factors for fetal alcohol spectrum disorders: Not as simple as it might seem. Alcohol Research \& Health, 34, 5-26.

May, P. A., Gossage, J. P., Marais, A. S., Adnams, C. M., Hoyme, H. E., Jones, K. L., \& Viljoen, D. L. (2007). The epidemiology of fetal alcohol syndrome and partial FAS in a South African community. Drug and Alcohol Dependence, 88, 259-271.

May, P. A., Miller, J. H., Goodhart, K. A., Maestas, O. R., Buckley, D., Trujillo, P. M., \& Gossage J. P. (2008). Enhanced case management to prevent fetal alcohol spectrum disorders in northern plains communities. Maternal and Child Health Journal, 12, 747-759.

Meyers, R. J., \& Smith, J. E. (1995). Clinical guide to alcohol treatment: The community reinforcement approach. New York, NY, United States: Guilford Press.

Meyers, R. J., Smith, J. E., \& Lash, D. (2003). The community reinforcement approach. In M. Galanter (Ed.), Recent developments in alcoholism. Research on alcoholism (Vol. XVI, pp.183-195). New York, NY, United States: Kluwer/Plenum.

Miller, W. R., Meyers, R. J., \& Hiller-Sturmhofel, S. (1999). The community-reinforcement approach. Alcohol Research \& Health, 23, 116-121.

Miller, W. R., \& Rollnick, S. (2002). Motivational interviewing. New York, United States: Guilford Press.

Miller, W. R., \& Rollnick, S. (2009). Ten things that motivational interviewing is not. Behavioural and Cognitive Psychotherapy, 37, 129-140.

Rapp, C. A., \& Goscha, R. J. (2004). The principles of effective case management of mental health services. Psychiatric Rehabilitation Journal, 27, 319-333.

Roozen, H. G., Boulogne, J. J., van Tulder, M. W., van den Brink, W., De Jong, C. A., \& Kerkhof, A. J. (2004). A systematic review of the effectiveness of the community reinforcement approach in alcohol, cocaine and opioid addiction. Drug and Alcohol Dependence, 74, 1-13.

Schneidman, E. S. (1999). The Psychological Pain Assessment Scale. Suicide and Life-Threatening Behavior, 29, 287-294.

SPSS 19.0 (2010). Command Syntax Reference. Chicago, IL, United States: SPSS Inc.

Stratton , K., Howe, C., \& Battaglia , F. (Eds.). (1996). Fetal Alcohol Syndrome: Diagnosis, epidemiology, prevention, and treatment. Washington, DC, United States: National Academy Press.

Timberlake, E. M., Farber, M. Z., \& Sabatino, C. A. (2002). The general method of social work practice ( $4^{\text {th }}$ ed.). Boston, MA, United States: Allyn and Bacon.

Urban, M., Chersich, M. F., Fourie, L. A., Chetty, C., Olivier, L., \& Viljoen, D. (2008). Fetal alcohol syndrome among grade 1 schoolchildren in Northern Cape Province: Prevalence and risk factors. South Africa Medical Journal, 98, 877-882.

Velasquez, M. M., Ingersoll, K. S., Sobell, M .B., Floyd, R. L., Sobell, L. C., \& von Sternberg, K. (2010). A dualfocus motivational intervention to reduce the risk of alcohol-exposed pregnancy. Cognitive and Behavioral Practice, 17, 203-212. doi:10.1016/j.cbpra.2009. 02004.

Viljoen, D. L., Gossage, J. P., Adnams, C., Jones, K. L., Robinson, L. K., Hoyme, H. E., . . May, P. A. (2005). Fetal Alcohol Syndrome epidemiology in a South African community: A second study of a very high prevalence area. Journal of Studies on Alcohol, 66, 593-604. 